\title{
Etiology and antibiotic susceptibility of bacterial pathogens responsible for community-acquired urinary tract infections in Poland
}

\author{
E. Stefaniuk ${ }^{1}$ - U. Suchocka ${ }^{2,3} \cdot$ K. Bosacka ${ }^{4}$ - W. Hryniewicz ${ }^{1}$
}

Received: 12 April 2016 / Accepted: 5 May 2016/Published online: 18 May 2016

(C) The Author(s) 2016. This article is published with open access at Springerlink.com

\begin{abstract}
Urinary tract infections (UTIs) are some of the most common infections in both community and hospital settings infections. With their high rate of incidence, recurrence, complications, diverse etiologic agents, as well as growing antibiotic resistance, UTIs have proven to be a serious challenge for medical professionals. The aim of this study was to obtain data on the susceptibility patterns of pathogens responsible for UTIs in Poland to currently used antibiotics. A total of 396 bacterial isolates were collected between March and May 2013 from 41 centers in all regions of Poland. The majority of isolates were from adult patients $(96.2 \%) ; 144$ (37.8 \%) patients were diagnosed with uncomplicated UTI, while the remaining $237(62.2 \%)$ had a complicated infection. The most prevalent pathogen was Escherichia coli (71.4 \%), followed by Klebsiella spp. (10.8\%) and the Proteae group (7.6\%). Escherichia coli was responsible for $80.6 \%$ of cases of uncomplicated and $65.8 \%$ of complicated infections. Only $65.8 \%$ of $E$. coli isolates were susceptible to ciprofloxacin (uncomplicated $75.9 \%$, complicated $58.3 \%$ ), $64.0 \%$ to
\end{abstract}

E. Stefaniuk

estefaniuk@cls.edu.pl

1 Department of Epidemiology and Clinical Microbiology, National Medicines Institute, Chełmska 30/34, 00-725 Warsaw, Poland

2 Faculty of Agriculture and Biology, Warsaw University of Life Sciences, Nowoursynowska 166, 02-787 Warsaw, Poland

3 Present address: Military Preventive Medicine Center in Cracow, Odrowąża 7, 30-901 Cracow, Poland

4 Centre of Quality Control in Microbiology, Rydygiera 8 (20A), 01-793 Warsaw, Poland nitrofurantoin $(67.2 \%, 62.8 \%), 65.1 \%$ to trimethoprim/ sulfamethoxazole $(68.1 \%, 62.8 \%)$, and $66.4 \%$ to fosfomycin (77.6\%, 62.2\%). Among E. coli isolates from all UTIs, only $43.4 \%$ were susceptible to ampicillin, with $47.4 \%$ from uncomplicated compared with $40.4 \%$ from complicated infections; $88.2 \%$ to amoxicillin/clavulanic acid $(91.4 \%$ vs. $85.9 \%$ complicated); $90.1 \%$ to cefuroxime $(93.1 \%$, $87.8 \%$ ); and $94.1 \%$ to cefotaxime $(98.2 \%, 91.0 \%)$. Thirty-five strains $(10.4 \%)$ were capable of producing extended-spectrum $\beta$-lactamases (ESBLs). This study demonstrates an increase in multidrug-resistant strains, especially among the leading pathogens associated with UTIs, including E. coli, Klebsiella spp., and Proteus spp.

\section{Introduction}

Urinary tract infections (UTIs) are some of the most common infections in both community and hospital settings. With their high rate of incidence, recurrence, complications, diverse etiologic agents, as well as growing antibiotic resistance, UTIs have proven to be a serious challenge for medical professionals. They account for $10-20 \%$ of all infections treated in primary care and $30-40 \%$ of infections treated in hospitals.

Rapidly increasing bacterial resistance to antibiotics resulting in significantly limited treatment options and multidrug resistance is a growing concern. Therefore, knowledge of the current antibiotic susceptibility patterns of the major bacterial pathogens responsible for UTIs is important in order to permit the optimal choice for empiric treatment. The aim of this study is to provide the most up-to-date data on the epidemiology, etiology, and susceptibility patterns of major pathogens responsible for community-acquired UTIs in Poland. In addition, clinically important mechanisms of resistance and risk factors in patients predisposing to UTIs were analyzed. 


\section{Materials and methods}

The study was performed in the Department of Epidemiology and Clinical Microbiology (DECM) of the National Medicines Institute in Warsaw on a total of 396 bacterial isolates collected between March and May 2013 from 41 centers, representing all regions of Poland. The isolates were recovered from ambulatory patients with clinical symptoms of UTI and significant bacteriuria $[1,2]$. One isolate per patient was included in the study. The patients' demographic and epidemiological data were collected and analyzed.

\section{Identification}

All strains were reidentified by using ID GP and ID GN cards in VITEK Compact (bioMérieux, Marcy l'Etoile, France) and stored at $-70{ }^{\circ} \mathrm{C}$ in TSB broth supplemented (with blood for streptococci) and $10 \%$ glycerol. Before performing further studies, the strains were unfrozen and subcultured twice on Columbia agar plates with $5 \%$ sheep blood (Bio-Rad, Marnes-la-Coquette, France).

\section{Antimicrobial susceptibility}

Susceptibility to a range of antibiotics was evaluated by the disk diffusion method on Mueller-Hinton agar (MHA; BioRad, Marnes-la-Coquette, France) for Gram-negative rods, staphylococci, and enterococci, and MHA supplemented with $5 \%$ defibrinated horse blood and $20 \mathrm{mg} / \mathrm{L}$ NAD (Bio-Rad, Marnes-la-Coquette, France) for streptococci. Reading of the disk diffusion susceptibility results was performed automatically using the ADAGIO apparatus (Bio-Rad, Marnes-laCoquette, France).

The following disks for susceptibility testing were used: ampicillin $(10 \mu \mathrm{g})$, amoxicillin/clavulanic acid $(20 \mu \mathrm{g} /$ $10 \mu \mathrm{g})$, amikacin $(30 \mu \mathrm{g})$, benzylpenicillin (1 U), cefalexin $(30 \mu \mathrm{g})$, cefepime $(30 \mu \mathrm{g})$, cefotaxime $(30 \mu \mathrm{g})$, ceftazidime $(30 \mu \mathrm{g})$, cefuroxime $(30 \mu \mathrm{g})$, ciprofloxacin $(5 \mu \mathrm{g})$, erythromycin $(15 \mu \mathrm{g})$, gentamicin $(10 \mu \mathrm{g})$, gentamicin $(30 \mu \mathrm{g})$, imipenem $(10 \mu \mathrm{g})$, clindamycin $(2 \mu \mathrm{g})$, meropenem $(10 \mu \mathrm{g})$, nitrofurantoin $(100 \mu \mathrm{g})$, piperacillin/tazobactam $(100 \mu \mathrm{g} /$ $10 \mu \mathrm{g})$, teicoplanin $(30 \mu \mathrm{g})$, trimethoprim/sulfamethoxazole $(11.25 \mu \mathrm{g} / 23.75 \mu \mathrm{g})$, fosfomycin $(200 \mu \mathrm{g})$, vancomycin $(5 \mu \mathrm{g})$ (Bio-Rad, Marnes-la-Coquette, France). Minimum inhibitory concentrations (MICs) were determined by VITEK Compact (bioMérieux, Marcy l'Etoile, France) and, in addition, by antibiotic gradient strips, Etest (bioMérieux, Marcy l'Etoile, France), and MICE (Oxoid Limited, Hampshire, United Kingdom), for extended-spectrum $\beta$-lactamase (ESBL)-producing strains.

The interpretation of susceptibility results was performed according to the European Committee on Antimicrobial Susceptibility Testing (EUCAST) guidelines [3], with the exception of fosfomycin, which was interpreted according to the Clinical and Laboratory Standards Institute (CLSI) guidelines [4].

ESBL production was detected by the double-disk synergy test (DDST) [5]. To detect genes encoding ESBLs, specific primers were used for polymerase chain reaction (PCR) assays. $\beta$-Lactamases were profiled by isoelectrofocusing, as described previously [6-8]. Total bacterial DNA was purified with a Genomic DNA Prep Plus kit (A\&A Biotechnology, Gdansk, Poland). PCR products were subjected to electrophoresis in a $1 \%$ agarose gel (Blirt, Gdansk, Poland) with ethidium bromide (Merck, Darmstadt, Germany).

High-level aminoglycoside resistance (HLAR) detection in enterococci was performed by disk diffusion with a $30-\mu \mathrm{g}$ gentamicin disk (Bio-Rad, Marnes-la-Coquette, France) [3].

\section{Quality control}

Escherichia coli ATCC 25922, E. coli ATCC 35218, Enterococcus faecalis ATCC 29212, Klebsiella pneumoniae ATCC 700603, Pseudomonas aeruginosa ATCC 27853, and Streptococcus pneumoniae ATCC 49619 from the collection of the National Medicines Institute in Warsaw were used as reference strains.

\section{Results}

The majority of isolates were from adult patients $(n=381$; $96.2 \%$ ) aged 19 to 94 years (average 60 years), with patients aged less than 40 years $(n=67)$ accounting for $17.6 \%$ of the total. As there were only 15 isolates (3.8\%) from children aged 1-18 years, these were excluded from further analysis. Among isolates from adults, more than $77.2 \%(n=294)$ were derived from females, and females predominated in all age groups. The majority of strains $(n=207 ; 54.3 \%)$ were derived from patients aged 65 years and over, of which 150 (72.5\%) were from females and $57(27.5 \%)$ were from males. One hundred and forty-four (37.8\%) patients were diagnosed with uncomplicated UTI, while the remaining 237 (62.2\%) had a complicated infection. The latter group comprised patients presenting with recurrent UTI $(n=126 ; 58.3 \%)$, urinary/ fecal incontinence $(n=67 ; 31.0 \%)$, nephritis $(n=51$; $23.6 \%)$, kidney stones $(n=46,21.3 \%)$, prostate hypertrophy $(n=12 ; 5.56 \%)$, renal insufficiency $(n=7 ; 3.2 \%)$, diabetes $(n=9 ; 4.17 \%)$, kidney empyema $(n=4 ; 1.85 \%)$, polycystic kidneys $(n=3 ; 1.39 \%)$, and kidney cancer $(n=3 ; 1.39 \%)$.

Aerobic Gram-negative bacteria were the dominant pathogens $(n=356 ; 93.4 \%)$. The majority $(n=347 ; 91.1 \%)$ of isolates belonged to the Enterobacteriaceae, while 9 (2.5\%) strains belonged to the non-fermenting rods. The most frequently isolated species from both uncomplicated and complicated infections were E. coli (71.4\%), followed by Klebsiella 
spp. $(10.8 \%)$ and the Proteae group (7.6\%), including Proteus mirabilis, P. vulgaris, Providencia rettgeri, and Morganella morganii in uncomplicated UTI. Escherichia coli was responsible for $80.6 \%$ of cases, compared with $65.8 \%$ of complicated cases. The proportion of Klebsiella spp. and Proteus spp. causing complicated UTIs was higher compared with those causing uncomplicated infections, accounting for $13.5 \%$ vs. $6.3 \%$ and $9.7 \%$ vs. $3.5 \%$, respectively. Escherichia coli was mainly responsible for UTIs in females ( $n=229 ; 77.9 \%)$, followed by Klebsiella spp. $(n=26 ; 8.8 \%)$ and Proteus spp. $(n=13 ; 4.4 \%)$. In females aged less than 65 years, E. coli accounted for $86.7 \%$ of uncomplicated and $74.7 \%$ of complicated infections $(p=0.34)$. Among females aged 65 years and over, E. coli was responsible for $72.1 \%$ of uncomplicated and $68.5 \%$ of complicated infections. It is interesting that, among females aged less than 30 years $(n=36)$, E. coli and E. faecalis were responsible for $88.9 \%$ and $11.1 \%$ of UTIs, respectively, with no other pathogen isolated.

Almost half of the isolates from UTI in men were $E$. coli ( $n=43 ; 49.4 \%$ ), followed by Klebsiella spp. ( $n=15 ; 17.2 \%)$. A higher proportion of Proteus spp. was observed in men compared to women, with $16.1 \%$ and $4.4 \%$, respectively $(p=0.01)$.

Susceptibility to antimicrobial agents was higher among isolates from uncomplicated than complicated infections (Table 1). Among E. coli isolates from all UTIs, only $43.4 \%$ were susceptible to ampicillin, with $47.4 \%$ from uncomplicated compared with $40.4 \%$ from complicated infections; $88.2 \%$ to amoxicillin/clavulanic acid (91.4\% uncomplicated, $85.9 \%$ complicated); $90.1 \%$ to cefuroxime $(93.1 \%, 87.8 \%)$; and $94.1 \%$ to cefotaxime $(98.2 \%, 91.0 \%)$. Only $65.8 \%$ of $E$. coli isolates were susceptible to ciprofloxacin (uncomplicated $75.9 \%$, complicated $58.3 \%$ ), $64.0 \%$ to nitrofurantoin (67.2\%, 62.8\%), $65.1 \%$ to trimethoprim/ sulfamethoxazole $(68.1 \%, 62.8 \%)$, and $66.4 \%$ to fosfomycin $(77.6 \%, 62.2 \%)$. Resistance to three or more drugs was higher among isolates recovered from complicated than uncomplicated infections $(41.0 \%$ vs. $13.8 \%$ ). The most common resistance phenotype comprised resistance to ampicillin, ciprofloxacin, and trimethoprim/sulfamethoxazole. Over $90 \%$ of $E$. coli isolates recovered from both types of infections were susceptible to aminoglycosides, third- and fourth-generation cephalosporins, and piperacillin/tazobactam, and all were susceptible to carbapenems.

In women aged 65 years or more with uncomplicated infections, susceptibility of $E$. coli to ciprofloxacin and trimethoprim/sulfamethoxazole was lower than in those aged less than 65 years $(88.9 \%$ vs. $54.6 \%$ and $76.4 \%$ vs. $54.6 \%$, respectively). In addition, differences were observed in the susceptibility of $E$. coli to trimethoprim/sulfamethoxazole and ciprofloxacin recovered from complicated infections in men versus women $(68.1 \%$ vs. $48.8 \%$ and $62.8 \%$ vs. $46.5 \%$, respectively).

Production of ESBLs was found in $2.8 \%$ of Enterobacteriaceae recovered from uncomplicated infections compared with $13.5 \%$ of isolates from complicated infections. Klebsiella pneumoniae was the pathogen most frequently associated with ESBL production, with $46.2 \%$ of all $K$. pneumoniae isolates being positive.

All ESBLs belonged to the CTX-M family and were classified on the basis of their reaction with specific primers, with 35 isolates belonging to CTX-M-1 and one to CTX-M-9.

Only nine non-fermenting Gram-negative rods were identified: seven Pseudomonas aeruginosa and two Acinetobacter baumannii. These were all recovered from patients with complicated UTI. Pseudomonas aeruginosa isolates were broadly susceptible to the key antibiotics tested, with $100 \%$ susceptibility to carbapenems, ciprofloxacin, piperacillin/tazobactam, and gentamicin. One of the $A$. baumannii strains was resistant to carbapenems.

Gram-positive cocci were isolated from 25 patients and accounted for $6.6 \%$ of all the isolates, with 20 E. faecalis and five $S$. agalactiae identified. The former were most frequently recovered from females $(n=13)$, while $S$. agalactiae were isolated exclusively from females. In $48 \%$ of cases, both of these pathogens were responsible for complicated infections. The E. faecalis isolates were fully susceptible to penicillin, ampicillin, nitrofurantoin, fosfomycin, and vancomycin; however, almost $35 \%$ presented with the HLAR phenotype. Streptococcus agalactiae was fully susceptible to the antibiotics tested.

\section{Discussion}

The data presented show that $E$. coli remains the most common etiologic agent of community-acquired UTI in Poland, although its role in etiology differs depending on the type of infection (uncomplicated vs. complicated) and the patient's characteristics. The overall percentage of E. coli as an etiologic agent of community-acquired UTIs in Poland decreased from $83.7 \%$ in our previous study published in 1998/99 to $71.4 \%$ in 2013 [9]. However, E. coli still predominates in uncomplicated UTIs $(80.6 \%)$ and even more so in younger females aged less than 30 years $(88.9 \%)$. A multicenter study (ARESC) on uncomplicated UTIs carried out in nine European countries and Brazil showed that E. coli was responsible for $76.7 \%$ of infections, ranging from $68.1 \%$ in Austria to $83.8 \%$ in France [10]. Among Polish isolates included in this study, E. coli was responsible for $75.6 \%$, which is somewhat lower than that revealed in the present investigation. 
Table 1 Susceptibility of all Enterobacteriaceae rods and Escherichia coli isolated from uncomplicated and complicated community-acquired urinary tract infections (UTIs) to various antimicrobial agents

\begin{tabular}{|c|c|c|c|c|c|c|}
\hline \multirow[t]{3}{*}{ Antimicrobials } & \multicolumn{2}{|c|}{ All cases of UTIs $(N=381)$} & \multicolumn{2}{|c|}{ Uncomplicated UTIs $(N=144)$} & \multicolumn{2}{|l|}{ Complicated UTIs $(N=237)$} \\
\hline & $\begin{array}{l}\text { Enterobacteriaceae } \\
\text { strains }(n=347)^{\mathrm{a}} \\
\text { Susceptible strains }\end{array}$ & $\begin{array}{l}\text { E. coli } \\
(n=272) \\
\text { n'; \%) }\end{array}$ & $\begin{array}{l}\text { Enterobacteriaceae strains } \\
(n=133)^{\mathrm{b}}\end{array}$ & $\begin{array}{l}\text { E. coli } \\
(n=116)\end{array}$ & $\begin{array}{l}\text { Enterobacteriaceae strains } \\
(n=214)^{\mathrm{c}}\end{array}$ & $\begin{array}{l}\text { E. coli } \\
(n=156)\end{array}$ \\
\hline & n' $(\%)$ & n' (\%) & n' (\%) & n' (\%) & n' (\%) & n' (\%) \\
\hline Ampicillin & $134(38.6 \%)$ & $118(43.4 \%)$ & $58(43.6 \%)$ & $55(47.4 \%)$ & $76(35.5 \%)$ & $63(40.4 \%)$ \\
\hline $\begin{array}{l}\text { Amoxicillin/clavulanic } \\
\text { acid }\end{array}$ & $295(84.0 \%)$ & $240(88.2 \%)$ & $120(90.2 \%)$ & $106(91.4 \%)$ & $175(81.8 \%)$ & $134(85.9 \%)$ \\
\hline Piperacillin/tazobactam & $310(89.3 \%)$ & $253(93.0 \%)$ & $131(98.5 \%)$ & $115(99.1 \%)$ & $179(83.6 \%)$ & $138(88.5 \%)$ \\
\hline Cefuroxime & $285(82.1 \%)$ & $245(90.1 \%)$ & $120(90.2 \%)$ & $108(93.1 \%)$ & $165(77.1 \%)$ & $137(87.8 \%)$ \\
\hline Cefotaxime & $300(86.5 \%)$ & $256(94.1 \%)$ & $128(96.2 \%)$ & $114(98.2 \%)$ & $172(80.4 \%)$ & $142(91.0 \%)$ \\
\hline Ceftazidime & $306(88.2 \%)$ & $259(95.2 \%)$ & $127(95.5 \%)$ & $114(98.2 \%)$ & $179(83.6 \%)$ & $145(93.0 \%)$ \\
\hline Cefixime & $310(89.3 \%)$ & $260(95.6 \%)$ & $128(96.2 \%)$ & $114(98.2 \%)$ & $183(85.5 \%)$ & $146(93.6 \%)$ \\
\hline Cefepime & $316(91.1 \%)$ & $264(97.1 \%)$ & $129(94.9 \%)$ & $115(99.1 \%)$ & $187(87.4 \%)$ & $149(95.5 \%)$ \\
\hline Imipenem & $344(99.1 \%)$ & $272(100 \%)$ & $131(98.5 \%)$ & $116(100 \%)$ & $213(99.5 \%)$ & $156(100 \%)$ \\
\hline Meropenem & $347(100 \%)$ & $272(100 \%)$ & $133(100 \%)$ & $116(100 \%)$ & $214(100 \%)$ & $156(100 \%)$ \\
\hline Amikacin & $333(96.0 \%)$ & $269(98.9 \%)$ & $132(99.3 \%)$ & $116(100 \%)$ & $201(93.9 \%)$ & $153(98.1 \%)$ \\
\hline Gentamicin & $308(88.8 \%)$ & $252(92.7 \%)$ & $124(93.2 \%)$ & $110(94.8 \%)$ & $184(86.0 \%)$ & $142(91.0 \%)$ \\
\hline Ciprofloxacin & $211(60.8 \%)$ & $179(65.8 \%)$ & $98(73.7 \%)$ & $97(75.9 \%)$ & $113(52.8 \%)$ & $91(58.3 \%)$ \\
\hline Nitrofurantoin & $194(55.9 \%)$ & $174(64.0 \%)$ & $81(60.9 \%)$ & $88(67.2 \%)$ & $113(52.8 \%)$ & $98(62.8 \%)$ \\
\hline Fosfomycin & nd & $188(66.4 \%)$ & nd & $90(77.6 \%)$ & nd & $97(62.2 \%)$ \\
\hline $\begin{array}{l}\text { Trimethoprim/ } \\
\text { sulfamethoxazole }\end{array}$ & $209(60.2 \%)$ & $177(65.1 \%)$ & $89(66.9 \%)$ & $79(68.1 \%)$ & $120(56.1 \%)$ & $98(62.8 \%)$ \\
\hline
\end{tabular}

nd, not determined

${ }^{a}$ Enterobacteriaceae strains responsible for UTIs: E. coli $(n=272)$, K. pneumoniae $(n=39)$, K. oxytoca $(n=2), P$. mirabilis $(n=25), P$. vulgaris $(n=2)$, P. rettgeri $(n=1), C$. freundii $(n=2), C$. koseri $(n=1)$, E. cloacae $(n=1), S$. marcescens $(n=1)$, M. morganii $(n=1)$

${ }^{\mathrm{b}}$ Enterobacteriaceae strains responsible for uncomplicated UTIs: E. coli $(n=116)$, K. pneumoniae $(n=9)$, P. mirabilis $(n=5)$, C. freundii $(n=1)$, C. $\operatorname{koseri}(n=1)$, M. morganii $(n=1)$

${ }^{\mathrm{c}}$ Enterobacteriaceae strains responsible for complicated UTIs: E. coli $(n=156)$, K. pneumoniae $(n=30)$, K. oxytoca $(n=2), P$. mirabilis $(n=20)$, $P$. vulgaris $(n=2), P$. rettgeri $(n=1), C$. freundii $(n=1)$, E. cloacae $(n=1), S$. marcescens $(n=1)$

In this study, we observed a decreasing percentage of E. coli among the Enterobacteriaceae responsible for complicated infections. In particular, we noted an increase in Klebsiella spp. from $2.8 \%$ in $1998 / 99$ to $10.8 \%$ in the current study, mainly in complicated infections, resulting in an increase in ESBL-positive strains and, thus, multidrugresistant isolates. By contrast, the percentage of Proteus spp. did not change significantly compared with the previous study [9]. Similar distributions of etiological agents causing UTI have been presented by other authors [10-17]. In the current study, over $46 \%$ of Klebsiella spp. were found to produce ESBLs and all but one of these isolates were recovered from complicated infections and all but one were found to belong to CTX-M group 1, which is the most common ESBL type in Poland [18]. A high prevalence of ESBL-producing Enterobacteriaceae has also been demonstrated in other studies $[11,15,19]$. A recent publication also indicates that ESBLproducing strains are more frequent in older patients [20], which is similar to the findings presented here.

From analyses of uncomplicated infections in the ARESC study [10], which included data from Poland, only a low percentage (5.6\%) of $K$. pneumoniae isolates producing ESBLs were identified, and this is comparable to the results obtained in the current paper. In our study, Proteus spp. was particularly frequent in males, accounting for more than $16.1 \%$ of cases compared with $5.3 \%$ in females, which was also reported in other publications $[17,20]$.

In general, Gram-positive pathogens are much less frequently isolated from UTIs than the Enterobacteriaceae and were responsible for $6.6 \%$ of all infections in this study and $5 \%$ in our previous study [9]. Similar data are also published from other countries $[15,21,22]$. In the present study, only E. faecalis and S. agalactiae were recovered, with no single isolate of $S$. saprophyticus. The lack of the isolation of the latter species may result from the fact that they are mainly responsible for uncomplicated infections in young women, who constituted only $19 \%(n=56)$ of our collection. This group of patients is usually given empiric treatment without taking a sample for culture $[10,16,21,23,24]$. Another reason for the lack of $S$. saprophyticus in our study collection could be due to the time of the year over which the study was conducted, i.e., winter, as it was previously observed in 
a previous study from Europe and the US that the prevalence of this pathogen varies depending on the time of the year observed [10, 21, 25]. In the ARESC study, S. saprophyticus was found to be responsible for only $4.2 \%$ of all cases of uncomplicated UTI [26].

Several recent studies have underlined an important change in resistance patterns among urinary pathogens. Since $E$. coli is the leading cause of UTI, an increase in resistance of this pathogen is of particular concern. Although the proportion of ESBL-producers among this pathogen and the other members of Enterobacteriaceae has not increased compared to our earlier study, the proportion of resistance to other antibiotics has increased substantially. The most notable changes among E. coli causing uncomplicated UTI cases have been observed for nitrofurantoin, ciprofloxacin, trimethoprim/sulfamethoxazole, and fosfomycin, with susceptibility to nitrofurantoin decreasing to $67.2 \%$ (from $91.2 \%$ in the previous study), susceptibility to ciprofloxacin decreasing to $73.7 \%$ (from $96.1 \%$ ), susceptibility to trimethoprim/sulfamethoxazole decreasing to $68.1 \%$ (from $80.9 \%$ ), and susceptibility to fosfomycin decreasing to $77.6 \%$ (from $96.7 \%$ ) [9]. Additionally, important differences in susceptibility were noticed between $E$. coli isolated from younger (aged less than 65 years) and older (aged 65 years and more) females in a study of uncomplicated infections, especially in terms of susceptibility to ciprofloxacin $(88.8 \%$ vs. $54.5 \%)$ and trimethoprim/sulfamethoxazole (76.4\% vs. $54.6 \%$ ). Similar results have been reported in a recent paper from Hong Kong [27]. This may be due to the fact that older females usually have more recurrent infections that were previously treated and could have had more contact with hospital settings.

Two recent studies have underlined that $E$. coli is isolated less frequently from males than from females and that isolates from complicated infections in males are much more resistant $[17,28]$. Our study confirmed this finding. The percentage of isolates susceptible to ciprofloxacin in E. coli strains from females and males were $62 \%$ and $46.5 \%$, respectively, and to trimethoprim/sulfamethoxazole were $68.1 \%$ and $48.8 \%$, respectively. Nitrofurantoin is one of the most frequently used drugs for treating uncomplicated UTIs, and resistance to this drug is generally very low, as indicated by numerous studies [29-31], including earlier data from Poland [9, 10, 22, 26]. A high proportion of resistance to this drug among E. coli as presented in this study is of concern. The only explanation for this finding could be the very high consumption of furazidin, a member of the same chemical group as nitrofurantoin, in Poland. This drug has been available without prescription in Poland since 2011. The sales have been increasing steadily year-on-year and its consumption as measured in defined daily doses (DDD) per 1000 population per day has increased from 2.13 in 2010 to 2.83 in 2012, an increase of $32.9 \%$. Looking at the susceptibility data of the isolates from complicated UTIs examined in this study, the treatment options for oral therapy are very limited, since less than $60 \%$ of $E$. coli were susceptible to ciprofloxacin and slightly over $63 \%$ were susceptible to trimethoprim/ sulfamethoxazole.

The increasing resistance among bacterial populations of urinary tract pathogens is particularly indicated by the growing number of multidrug-resistant strains. Multidrug resistance is defined as non-susceptibility to at least one agent in each of three or more antimicrobial groups [32]. Important differences between countries and regions with regard to the percentage of MDR among E. coli UTI isolates have been reported, ranging from $16.7 \%$ in Portugal to $34 \%$ in California, and as high as $76.5 \%$ in India [29, 33, 34]. In our study, multidrug-resistant $E$. coli isolated from complicated infections was found to be $41 \%$. The most common risk factors associated with multidrug-resistant $E$. coli, identified in almost $21 \%$ of patients in our study, were nephritis and other renal pathologies, while in a study from India, diabetes was determined to be one of the most common risk factors for multidrug resistant $E$. coli, in addition to renal pathologies [34].

Multidrug resistance is the most rapidly increasing resistance phenotype, particularly among $K$. pneumoniae, due to the efficient spread of ESBL-producing, and more recently carbapenemase-producing, strains [35-38]. Currently, such strains are mostly isolated from hospitalized patients, but their presence is now increasing rapidly in the community. In our study, $71.8 \%$ of $K$. pneumoniae presented with the multidrug resistance phenotype, with the most common resistance pattern including resistance to cefuroxime, ceftazidime, ciprofloxacin, and trimethoprim/sulfamethoxazole. Ongoing monitoring of resistance trends is of great importance.

\section{Conclusion}

This study presents the changing etiology of urinary tract infections (UTIs) in Poland over the past 14 years and demonstrates an increase in multidrug-resistant strains, especially among the leading pathogens associated with UTIs, including Escherichia coli, Klebsiella spp., and Proteus spp. It also highlights the importance of ongoing surveillance at the local level in order to guide empiric treatment recommendations for local use.

Acknowledgments We thank all the Polish microbiologists who participated in this study and Stephen Murchan for the critical reading of the manuscript. Part of this study was presented at the 25th European Congress of Clinical Microbiology and Infectious Diseases (ECCMID), Copenhagen, Denmark, 25-28th April 2015. 
Compliance with ethical standards Isolates were obtained as part of the routine activity of the Department of Epidemiology and Clinical Microbiology (DECM) and were analyzed anonymously in a retrospective manner. Ethical approval and informed consent were, thus, not required.

Funding This study was partially financed by the health program of the Polish Ministry of Health "Narodowy Program Ochrony Antybiotyków na lata 2011-2015" and by SPUB grant MIKROBANK from the Polish Ministry of Science and Higher Education.

Conflict of interest The authors declare that they have no conflict of interest.

Ethical approval The authors declare that ethical approval was not required.

Informed consent The authors declare that informed consent was not required.

Open Access This article is distributed under the terms of the Creative Commons Attribution 4.0 International License (http:// creativecommons.org/licenses/by/4.0/), which permits unrestricted use, distribution, and reproduction in any medium, provided you give appropriate credit to the original author(s) and the source, provide a link to the Creative Commons license, and indicate if changes were made.

\section{References}

1. Stamm WE (2008) Urinary tract infections, pyelonephritis, and prostatitis. In: Fauci AS, Kasper DL, Longo DL, Braunwald E, Hauser SL, Jameson JL, Loscalzo J (eds) Harrison's principles of internal medicine, 17th edn. McGraw-Hill, New York, pp 18201830

2. Hooton T (2010) Urinary tract infections in adults. In: Floege J, Johnson RJ, Feehally J (eds) Comprehensive clinical nephrology, 4th edn. Elsevier Saunders, St. Louis, pp 629-640

3. European Committee on Antimicrobial Susceptibility Testing (EUCAST) (2014) Breakpoint tables for interpretation of MICs and zone diameters. Version 4.0. Available online at: http://www. eucast.org/fileadmin/src/media/PDFs/EUCAST files/Breakpoint tables/Breakpoint_table_v_4.0.pdf. Accessed 17 Dec 2015

4. Clinical and Laboratory Standards Institute (CLSI) (2014) Performance standards for antimicrobial susceptibility testing; Twenty-fourth informational supplement. CLSI document M100S24. CLSI, Wayne, PA

5. Drieux L, Brossier F, Sougakoff W, Jarlier V (2008) Phenotypic detection of extended-spectrum $\beta$-lactamase production in Enterobacteriaceae: review and bench guide. Clin Microbiol Infect 14 (Suppl 1):90-103. doi:10.1111/j.1469-0691.2007.01846. $\mathrm{x}$

6. Sana T, Rami K, Racha B, Fouad D, Marcel A, Hassan M et al (2011) Detection of genes TEM, OXA, SHV and CTX-M in 73 clinical isolates of Escherichia coli producers of extended spectrum beta-lactamases and determination of their susceptibility to antibiotics. Int Arab J Antimicrob Agents 1:1-5

7. Pai H, Lyu S, Lee JH, Kim J, Kwon Y, Kim JW et al (1999) Survey of extended-spectrum $\beta$-lactamases in clinical isolates of Escherichia coli and Klebsiella pneumoniae: prevalence of TEM52 in Korea. J Clin Microbiol 37:1758-1763

8. Wang H, Kelkar S, Wu W, Chen M, Quinn JP (2003) Clinical Isolates of Enterobacteriaceae producing extended-spectrum $\beta$ lactamases: prevalence of CTX-M-3 at a hospital in China. Antimicrob Agents Chemother 47:790-793
9. Hryniewicz K, Szczypa K, Sulikowska A, Jankowski K, Betlejewska K, Hryniewicz W (2001) Antibiotic susceptibility of bacterial strains isolated from urinary tract infections in Poland. J Antimicrob Chemother 47:773-780

10. Schito GC, Naber KG, Botto H, Palou J, Mazzei T, Gualco L et al (2009) The ARESC study: an international survey on the antimicrobial resistance of pathogens involved in uncomplicated urinary tract infections. Int J Antimicrob Agents 34:407-413. doi:10.1016/ j.ijantimicag.2009.04.012

11. Wu YH, Chen PL, Hung YP, Ko WC (2014) Risk factors and clinical impact of levofloxacin or cefazolin nonsusceptibility or ESBL production among uropathogens in adults with communityonset urinary tract infections. J Microbiol Immunol Infect 47:197203. doi:10.1016/j.jmii.2012.09.001

12. Khawcharoenporn T, Vasoo S, Singh K (2013) Urinary tract infections due to multidrug-resistant Enterobacteriaceae: prevalence and risk factors in a Chicago Emergency Department. Emerg Med Int 2013:258517. doi:10.1155/2013/258517

13. Gordon KA, Jones RN; SENTRY Participant Groups (Europe, Latin America, North America) (2003) Susceptibility patterns of orally administered antimicrobials among urinary tract infection pathogens from hospitalized patients in North America: comparison report to Europe and Latin America. Results from the SENTRY Antimicrobial Surveillance Program (2000). Diagn Microbiol Infect Dis 45:295-301

14. Matthews SJ, Lancaster JW (2011) Urinary tract infections in the elderly population. Am J Geriatr Pharmacother 9(5):286-309. doi: 10.1016/j.amjopharm.2011.07.002

15. Maraki S, Mantadakis E, Michailidis L, Samonis G (2013) Changing antibiotic susceptibilities of community-acquired uropathogens in Greece, 2005-2010. J Microbiol Immunol Infect 46:202-209. doi:10.1016/j.jmii.2012.05.012

16. Katsarolis I, Poulakou G, Athanasia S, Kourea-Kremastinou J, Lambri N, Karaiskos E et al (2010) Acute uncomplicated cystitis: from surveillance data to a rationale for empirical treatment. Int $\mathrm{J}$ Antimicrob Agents 35:62-67. doi:10.1016/j.ijantimicag.2009.08. 018

17. Kiffer CRV, Mendes C, Oplustil CP, Sampaio JL (2007) Antibiotic resistance and trend of urinary pathogens in general outpatients from a major urban city. Int Braz J Urol 33:42-48

18. Literacka E, Baraniak A, Malinowska K, Herda M, Urbanowicz P, Bojarska K et al (2015) Prevalence of ESBLs, AmpCs and carbapenemases in Klebsiella pneumoniae clinical isolates in Poland. In: Proceedings of the 25th European Congress of Clinical Microbiology and Infectious Diseases (ECCMID), Copenhagen, Denmark, 25-28th April 2015, poster EV0096

19. Roxo I, Magalhães S, Ramalheira E, Ferreira S (2015) Epidemiology of ESBL-producing isolates causing UTI in the elderly (Aveiro, Portugal). In: Proceedings of the 25th European Congress of Clinical Microbiology and Infectious Diseases (ECCMID), Copenhagen, Denmark, 25-28th April 2015, poster PO974

20. Gopal Rao G, Batura D, Batura N, Nielsen PB (2015) Key demographic characteristics of patients with bacteriuria due to extended spectrum beta-lactamase (ESBL)-producing Enterobacteriaceae in a multiethnic community, in North West London. Infect Dis (Lond) 47:719-724. doi:10.3109/23744235.2015.1055588

21. Czaja CA, Scholes D, Hooton TM, Stamm WE (2007) Populationbased epidemiologic analysis of acute pyelonephritis. Clin Infect Dis 45:273-280

22. Naber KG, Schito G, Botto H, Palou J, Mazzei T (2008) Surveillance study in Europe and Brazil on clinical aspects and Antimicrobial Resistance Epidemiology in Females with Cystitis (ARESC): implications for empiric therapy. Eur Urol 54:11641175. doi:10.1016/j.eururo.2008.05.010 
23. Vincent CR, Thomas TL, Reyes L, White CL, Canales BK, Brown MB (2013) Symptoms and risk factors associated with first urinary tract infection in college age women: a prospective cohort study. J Urol 189:904-910. doi:10.1016/j.juro.2012.09.087

24. den Heijer CDJ, Donker GA, Maes J, Stobberingh EE (2010) Antibiotic susceptibility of unselected uropathogenic Escherichia coli from female Dutch general practice patients: a comparison of two surveys with a 5 year interval. J Antimicrob Chemother 65: 2128-2133. doi:10.1093/jac/dkq286

25. Raz R, Colodner R, Kunin CM (2005) Who are youStaphylococcus saprophyticus? Clin Infect Dis 40:896-898

26. Chlabicz S, Leszczyńska K, Lukas W, Gualco L, Schito G, Naber KG (2011) Uncomplicated lower urinary tract infections in females - clinical aspects, aetiology and antimicrobial resistance epidemiology. Results of the ARESC (Antimicrobial Resistance Epidemiological Survey on Cystitis) study in Poland and their implications for empiric therapy. Przegl Epidemiol 65:345-351

27. Ho PL, Wong RC, Yip KS, Loke SL, Leung MS, Mak GC et al (2007) Antimicrobial resistance in Escherichia coli outpatient urinary isolates from women: emerging multidrug resistance phenotypes. Diagn Microbiol Infect Dis 59:439-445

28. den Heijer CDJ, Penders J, Donker GA, Bruggeman CA, Stobberingh EE (2013) The importance of gender-stratified antibiotic resistance surveillance of unselected uropathogens: a Dutch Nationwide Extramural Surveillance study. PLoS One 8:e60497. doi:10.1371/journal.pone.0060497

29. Kahlmeter G, Poulsen HO (2012) Antimicrobial susceptibility of Escherichia coli from community-acquired urinary tract infections in Europe: the ECO·SENS study revisited. Int J Antimicrob Agents 39:45-51. doi:10.1016/j.jjantimicag.2011.09.013

30. Kamenski G, Wagner G, Zehetmayer S, Fink W, Spiegel W, Hoffmann K (2012) Antibacterial resistances in uncomplicated urinary tract infections in women: ECO.SENS II data from primary health care in Austria. BMC Infect Dis 12:222. doi:10.1186/14712334-12-222
31. Kahlmeter G, Åhman J, Matuschek E (2015) Antimicrobial resistance of Escherichia coli causing uncomplicated urinary tract infections: a European update for 2014 and comparison with 2000 and 2008. Infect Dis Ther 4:417-423. doi:10. 1007/s40121-015-0095-5

32. Magiorakos AP, Srinivasan A, Carey RB, Carmeli Y, Falagas ME, Giske CG et al (2012) Multidrug-resistant, extensively drugresistant and pandrug-resistant bacteria: an international expert proposal for interim standard definitions for acquired resistance. Clin Microbiol Infect 18:268-281. doi:10.1111/j.1469-0691.2011. 03570.x

33. Smith SP, Manges AR, Riley LW (2008) Temporal changes in the prevalence of community-acquired antimicrobial-resistant urinary tract infection affected by Escherichia coli clonal group composition. Clin Infect Dis 46:689-695. doi:10.1086/527386

34. Niranjan V, Malini A (2014) Antimicrobial resistance pattern in Escherichia coli causing urinary tract infection among inpatients. Indian J Med Res 139:945-948

35. Rampure R, Gangane R, Oli AK, Chandrakanth K (2013) Prevalence of MDR-ESBL producing Klebsiella pneumoniae isolated from clinical samples. J Microbiol Biotechnol Res 3:32-39

36. Ullah F, Malik SA, Ahmed J (2009) Antimicrobial susceptibility pattern and ESBL prevalence in Klebsiella pneumoniae from urinary tract infections in the North-West of Pakistan. Afr J Microbiol Res 3:676-680

37. Langarizadeh N, Ahangarzadeh RM, Aghazadeh M, Hasani A (2011) Prevalence of multi-drug resistant (MDR) Klebsiella pneumoniae among children and adults with urinary tract infection referred to tabriz teaching hospitals. Q J Biol Sci 4:9-17

38. Hyle EP, Lipoworth AD, Zaoutis TE, Nachamkin I, Fishman NO, Bilker WB et al (2005) Risk factors for increasing multidrug resistance among extended-spectrum $\beta$-lactamase-producing Escherichia coli and Klebsiella species. Clin Infect Dis 40:13171324 